\title{
Prevalencia de maloclusión en relación con hábitos de succión no nutritivos en niños de 3 a 9 años en Ferrol
}

\section{Prevalence of malocclusion in order to non nutritive sucking habits in children between 3 and 12 years-old in Ferrol}

\author{
Pipa Vallejo A*, Cuerpo García de los Reyes P**, López-Arranz Monje E***, \\ González García M****, Pipa Muñiz I*****, Acevedo Prado A******
}

\section{RESUMEN}

Se realizó un estudio observacional descriptivo entre octubre del 2008 y abril del 2009. Se seleccionaron 368 niños de 3 a 9 años del área sanitaria de Ferrol, con el objetivo de identificar la prevalencia de maloclusión en relación con los hábitos de succión no nutritivos (HSNN), (digital, chupete, biberón) y respiración oral.

También se realiza una comparación con el resto de estudios sobre el mismo tema, para poder saber cómo estamos actuando, desde el punto de vista preventivo, en la información sobre las consecuencias nocivas de los hábitos de succión no nutritivos en el desarrollo de una buena oclusión dental.

Palabras clave: Maloclusión, succión digital, resalte, hábitos de succión no nutritivos (HSNN), prevalencia.

\section{SUMMARY}

A descriptive observational study was carried out between October 2008 and April 2009. 368 children between 3 and 12 years-old were selected in Ferrol Health Board in order to identify the prevalence of the malocclusion related to the following non nutritive oral habits: thumb sucking, dummy and mouth breathing. In addition, a comparison with other studies about the same theme is carried out in order to establish how we are acting, from the preventative point of view, on the information about the harmful consequences of the non nutritive habits in the development of a good dental occlusion.

Key words: Maloclussion, thumb sucking, overjet, non nutritive sucking habits, (NNSH), prevalence.

Fecha de recepción: Diciembre de 2010.

Aceptado para publicación: Enero de 2011.

* $\quad$ Profesor Asociado. Escuela de Odontología. Facultad de Medicina. Universidad de Oviedo.

** Licenciada en Odontología. Práctica privada.

*** Profesora Asociada. Escuela de Odontología. Facultad de Medicina. Universidad de Oviedo.

**** Profesor Asociado. Servicio de Cirugía Oral y Maxilofacial del HUCA.

***** Médico Interno Residente. Hospital de Cabueñes.

******* Médico Técnico en Salud. Ferrol.

Pipa Vallejo A, Cuerpo García de los Reyes P, López-Arranz Monje E, González García M, Pipa Muñiz I, Acevedo Prado A. Prevalencia de maloclusión en relación con hábitos de succión no nutritivos en niños de 3 a 9 años en Ferrol. Av. Odontoestomatol 2011; 27 (3): 137-145. 


\section{INTRODUCCIÓN}

La oclusión hace referencia a las relaciones que se establecen al poner los arcos dentarios en contacto. También implica el análisis de cualquier relación de contacto entre los dientes: relaciones de protrusión, en lateralidad o céntrica. Según Angle, sería las relaciones normales de los planos inclinados de los dientes cuando las arcadas dentarias están en íntimo contacto.

La normal es aquella que es equilibrada y permite cumplir la función masticatoria y preservar la integridad de la dentición a lo largo de la vida en armonía con el conjunto estructural del aparato estomatognático.

El término maloclusión es genérico y debe aplicarse, sobre todo, a aquellas situaciones que exigen intervención ortodóncica más que a cualquier desviación de la oclusión ideal, ya que existe una línea continua entre lo ideal, lo normal y lo maloclusivo.

Ello ocurre con tanta frecuencia que la gran mayoría de maloclusiones conlleva una tendencia constitucional que va a ser potenciada o aliviada por factores ambientales múltiples (1).

El hábito es la costumbre o práctica adquirida por la repetición frecuente de un mismo acto. Los hay que se consideran fisiológicos, como son la respiración nasal, masticación, deglución y existen también aquellos no fisiológicos entre los cuales tenemos las succión no nutritiva (que puede ser de dedo, chupete, etc.), la respiración oral, la interposición lingual, etc. Los malos hábitos pueden alterar el normal desarrollo del sistema estomatognático produciendo un desequilibrio entre las fuerzas musculares externas e internas, ya que si una no ejerce su presión normal, permite que otra en su intensidad habitual produzca una deformación, dental u ósea. Otras veces se suman fuerzas que normalmente no están presentes, como la ejercida por un dedo o chupete. Los hábitos orales anómalos modifican la posición de los dientes y la relación y la forma que guardan las arcadas dentarias entre sí, interfiriendo en el crecimiento normal y en la función de la musculatura orofacial.

En el desarrollo de la maloclusión juega un papel importante el factor genético en el crecimiento cráneo facial y en el patrón oclusal básico.
El crecimiento maxilofacial está determinado genéticamente, pero en su desarrollo también influyen factores como función, oclusión, articulación, postura craneal y se define la triada morfología-función-postura como el trípode oral (2).

\section{Definición y clasificación de los HSNN}

1. La succión digital comienza en la vida fetal $\left(29^{a}\right.$ semana de la gestación), es normal al principio de la vida, en recién nacidos y durante los primeros meses. Es un comportamiento innato que se transforma en un hábito, se cree que cuando el niño está aburrido, ansioso o cansado es cuando persiste la succión digital.

Existen diferentes posiciones del dedo, normalmente el pulgar, en dicha succión; la más usual (50\%) consiste en introducir el pulgar profundamente, tocando el paladar plenamente y los incisivos inferiores están en contacto en su borde incisal con el nudillo del pulgar (3).

Para la mayoría de los autores la succión digital es una de las situaciones más nocivas durante el desarrollo de la oclusión normal $(4,5)$.

2. La succión del chupete empieza sobre los primeros 6 meses de vida. Es el hábito de succión no nutritiva más frecuente.

Hay mucha controversia sobre la edad a la que se debe quitar el chupete. Hay estudios que sugieren que es posible disminuir el peligro de estas malposiciones pidiéndoles a los padres que reduzcan el tiempo de chupete al niño, ya que al cesar el hábito, con menos de tres años, se curan espontáneamente (6).

\section{Consecuencias de los HSNN en el macizo estomatognático}

Si los HSNN se alargan en el tiempo podemos observar: paladar ojival, prognatismo maxilar, retrognatia mandibular, incisivos superiores vestibulizados e inferiores lingualizados, resalte aumentado, mordida abierta anterior o lateral, mordida cruzada posterior, tendencia a clase $2 / \operatorname{div} 1^{\text {a }}$, hipotonía labial, labio inferior hiperactivo con contracción anormal en la succión y deglución, 
También se puede observar: boca abierta, facilitando la respiración oral, persistencia de deglución infantil, problemas fonéticos, interposición lingual en reposo, alteraciones en el dedo, labio inferior colocado por detrás de los incisivos superiores, interferencia de la secuencia normal de la erupción, etc.

\section{OBJETIVO}

- Calcular la prevalencia de maloclusiones dentales relacionadas con los HSNN.

- Calcular los HSNN en nuestra área sanitaria.

- Identificar las distintas agrupaciones existentes en nuestra muestra de las distintas maloclusiones (resalte aumentado, mordida abierta anterior y mordida cruzada posterior).

- Determinar la significación estadística existente entre la presencia o no de los distintos HSNN estudiados y la presencia o no de las patologías analizadas (resalte aumentado, mordida abierta anterior y mordida cruzada posterior).

\section{MATERIAL Y MÉTODOS}

\section{Marco del estudio}

Este estudio se desarrolla en el área sanitaria de Ferrol entre los meses de octubre del 2008 y abril del 2009.

Dicha área comprende los ayuntamientos de Ares, Cabanas, A Capela, Cariño, Cedeira, Cercido, Fene, Ferrol, Mañón, Moeche, Monfero, Mugardos, Narón, Neda, Ortigueira, Pontedeume, As Pontes, San Sadurniño, Somozas y Valdoviño, con una superficie de $1.554,20 \mathrm{Km}^{2}$. Su población total es de 205.456 habitantes, de los cuales $20.815(10,1 \%)$ son niños entre 0 y 14 años, 10.622 son varones y 10.193 son mujeres.

\section{Sujetos del estudio}

Se seleccionaron varones y mujeres del área de Ferrol cuyos padres accedieron a colaborar. El rango de edad se estableció entre los 3 y 9 años porque en esos años aún se puede actuar sobre el hábito para que desaparezca, siendo el chupete y la succión hábitos de los niños más pequeños y la respiración oral de los niños mayores.

El muestreo se realizó de dos maneras: por una parte se requirió la colaboración de varios colegios: Cruceiro de Canido e Ibáñez Martín de Ferrol, Centieiras de Fene, San Sadurniño, Ortigueira, Valdoviño y Moeche. Asimismo en una clínica privada se revisó a los niños cuyos padres permitían participar en el proyecto y también contestaron a las preguntas sobre los hábitos no nutritivos de su hijo. El total son 161 (43,8\%) varones y 207 (56,3\%) mujeres.

\section{Criterios de inclusión:}

- Edad comprendida entre los 3-9 años y cuyos padres hayan dado su consentimiento por escrito.

- Raza latina para no tener influencias de patrones genéticos inherentes a otras razas.

\section{Criterios de exclusión:}

- Síndromes o retrasos mentales por su gran prevalencia de malformaciones en el macizo craneofacial.

- Ausencia de relación incisal a consecuencia del recambio dentario en dentición mixta.

\section{Análisis estadístico}

Para el análisis estadístico de los datos del estudio se utilizó el paquete estadístico SPSS 15.0 (SPSS Inc, Chicago, IL).

Para la comparación de variables categóricas se utilizó un análisis $\chi^{2}$. Para la comparación de variables cuantitativas se realizó un análisis con la $t$ de Student cuando la distribución de las mismas era normal. La comprobación de normalidad se realizó mediante un test de Kolmogorov-Smirnoff.

Se realizó un estudio descriptivo de la muestra así como de las principales variables relacionadas con la intervención y las variables principales del estudio.

Los valores se expresan como media y desviación típica para las variables continuas o como un porcentaje sobre el total del grupo para las variables categóricas. 
Se consideraron valores estadísticamente significativos aquellos cuyo valor de p fuese menor de 0,05.

\section{Metodología clínica}

En la revisión se anotaba la edad de inicio y fin del uso del chupete, así como la frecuencia de su uso (todo el día, solamente por la noche y al dormir), lo mismo con la succión digital si existía o había existido y se les preguntaba a los padres por dónde creían que respiraban sus hijos. Después de apuntar los datos con respecto a los HSNN se hacía una revisión de la boca del niño utilizando espejos, sondas y pinzas y secando los dientes con algodones para tener buena visibilidad. Se anotaba el tipo de respiración, la mordida abierta anterior, cruzada posterior y se medía el resalte.

\section{Variables del estudio}

El resalte, la mordida cruzada posterior, mordida abierta anterior, lateral, respiración nasal u oral.

\section{RESULTADOS}

El número de casos estudiados ha sido de 368. La distribución por sexos corresponde a 161 varones $(43,8 \%)$ y 207 mujeres (56,3\%) (Gráfico 1$)$.

La media de edad es de 6,3 años: 6,45 en mujeres (6,45 DT) y 5,89 en hombres (5,89 DT). Es estadís-

\section{Distribución por sexos de la muestra}
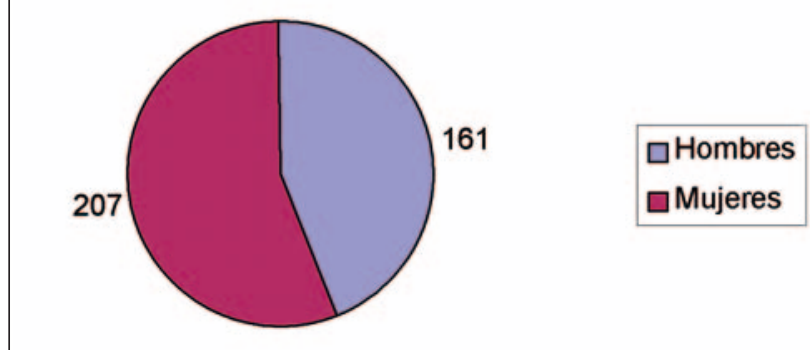

Gráfico 1. ticamente significativa $(p<0,05)$ la diferencia existente entre la edad de ambos sexos.

El número de pacientes que presentan maloclusión es de 139, siendo la prevalencia general de maloclusión (casos que presentan al menos un tipo de maloclusión) del 37,71\%. La distribución de las maloclusiones ordenados de menor a mayor son:

- La mordida abierta se presenta en un 12,5\% (el $63 \%$ son mujeres y el $37 \%$ hombres).

- La mordida cruzada en un $17,9 \%$ (el $60,9 \%$ son mujeres y el $39,1 \%$ son hombres).

- El resalte aumentado en un $28,8 \%$ (el 58,5\% son mujeres y el $41,5 \%$ son hombres).

La prevalencia de maloclusión en el grupo con chupete más de 3 años es la siguiente:

- La mordida abierta la presentan 16 casos (22,5\%), (13,5\% en mujeres y $11,2 \%$ en hombres). La diferencia observada es estadísticamente significativa $(\mathrm{p}<0,05)$.

- La mordida cruzada en un $23,9 \%$ (20,3\% en mujeres y $14,9 \%$ en hombres).

- El resalte aumentado en un 39,4\% (30\% de las mujeres y el $27,3 \%$ de los hombres).

Existe diferencia estadísticamente significativa $(p<0,05)$. entre la maloclusión hallada en el grupo global y la hallada en el grupo de casos con chupete más de 3 años.

La prevalencia de maloclusión en el grupo que presenta succión digital y/o chupete más de 3 años es del $45,9 \%$ :

- La mordida abierta se presenta en 25 casos, siendo un $25,5 \%$ de prevalencia.

- La mordida cruzada se presenta en 21 casos, siendo un $21,4 \%$ de prevalencia.

- El resalte aumentado se presenta en 40 casos, siendo un $40,8 \%$ de prevalencia.

- La respiración oral observada se presenta en 42 casos, siendo un 42,9\% de prevalencia (Gráfico 2).

Hay 24 casos $(6,5 \%)$ que presentan las tres patologías. Son 14 mujeres $(58,5 \%)$ y 10 hombres $(41,7 \%)$.

El hábito más frecuente de los analizados en este estudio es el chupete con una frecuencia del $83,7 \%$. 


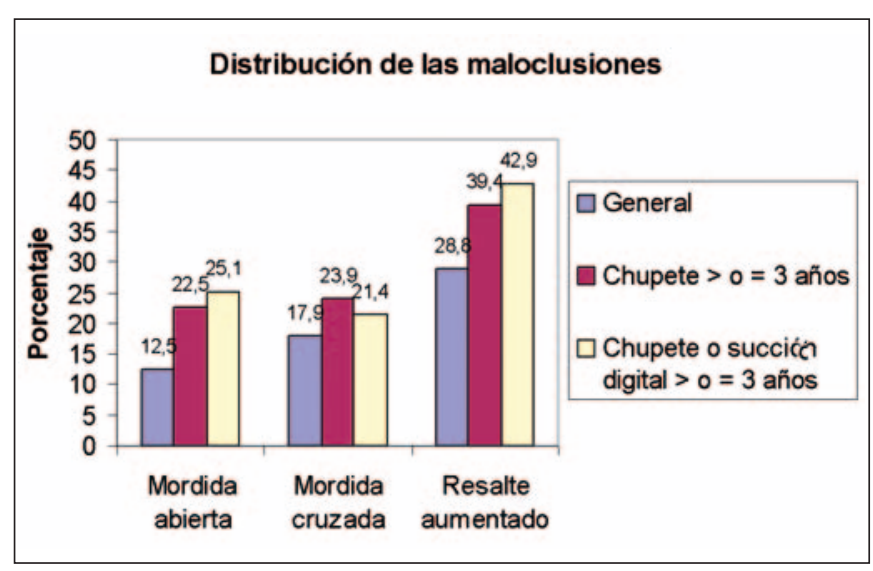

Gráfico 2.

A continuación se presenta la respiración oral observada en la revisión con una frecuencia del $41,6 \%$ (frente a un $22,6 \%$ de respiración oral detectada por los padres) y por último succión la digital con un $9 \%$ (Gráfico 3).

Con respecto a la distribución del uso del chupete:

- 60 casos $(16,3 \%)$ nunca lo han usado.

- 231 casos $(62,8 \%)$ lo usaron durante menos de tres años. El 59,1\% son mujeres.

- 77 casos (21\%) lo usaron 3 años o más. El 53,5\% son mujeres (Gráfico 4).

En cuanto a la frecuencia de la succión digital: 33 casos presentan succión digital (60,6\% mujeres y 39,4\% hombres) de los cuales 28 casos (85\%) continúan más de 3 años (67,9\% mujeres y 32,1\% hombres).

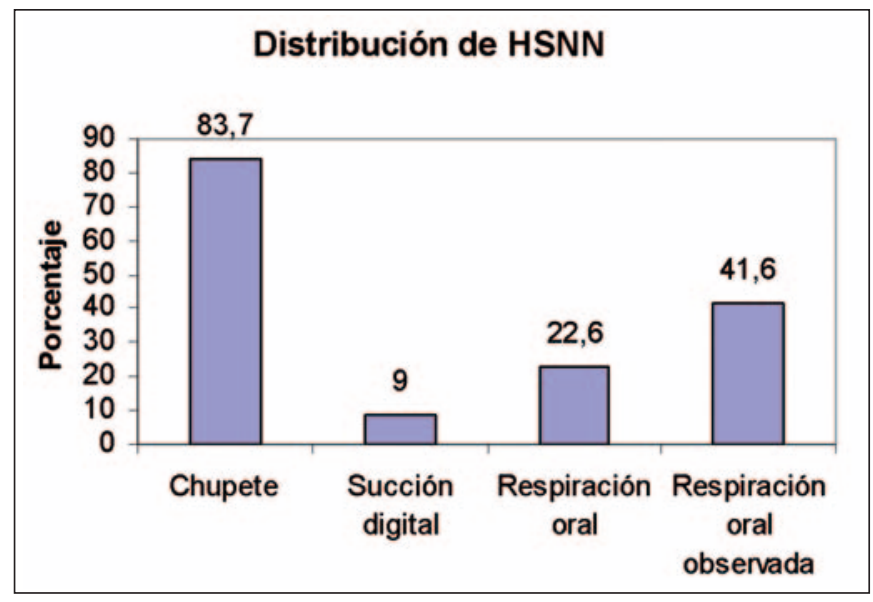

Gráfico 3.

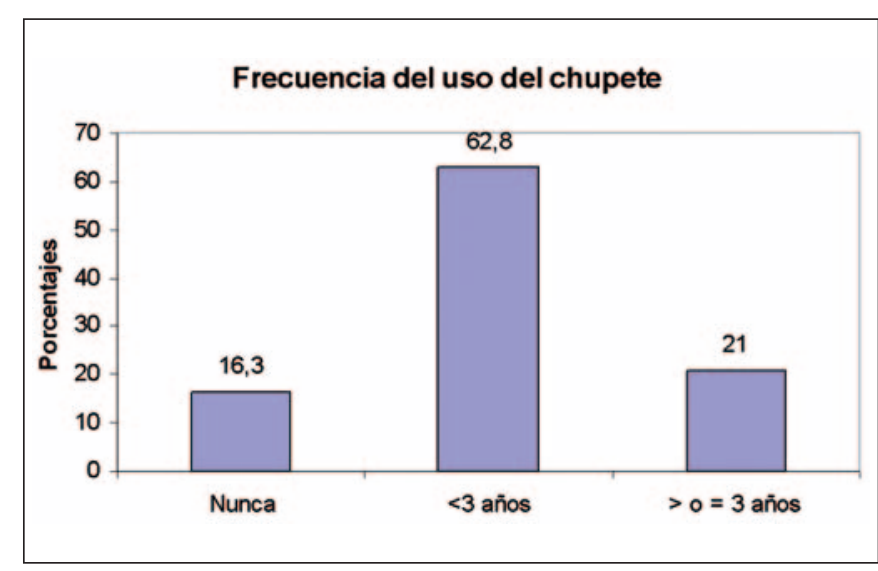

Gráfico 4.

El papel de la respiración oral es muy importante:

- El 28,1\% de los casos con respiración oral observada presentan mordida cruzada posterior frente al $10,7 \%$ de los pacientes sin respiración observada que presentan dicha patología.

- El 20,3\% de los casos con mordida abierta presentan respiración oral observada, sin embargo el $7 \%$ de los casos sin respiración oral observada tienen mordida abierta.

- El 40,5\% de los casos con resalte aumentado presentan respiración oral observada, en comparación con el $20,5 \%$ que presentan resalte aumentado sin respiración oral observada. Las diferencias anteriormente observadas son estadísticamente significativas $(p<0,05)$. (Gráfico 5).

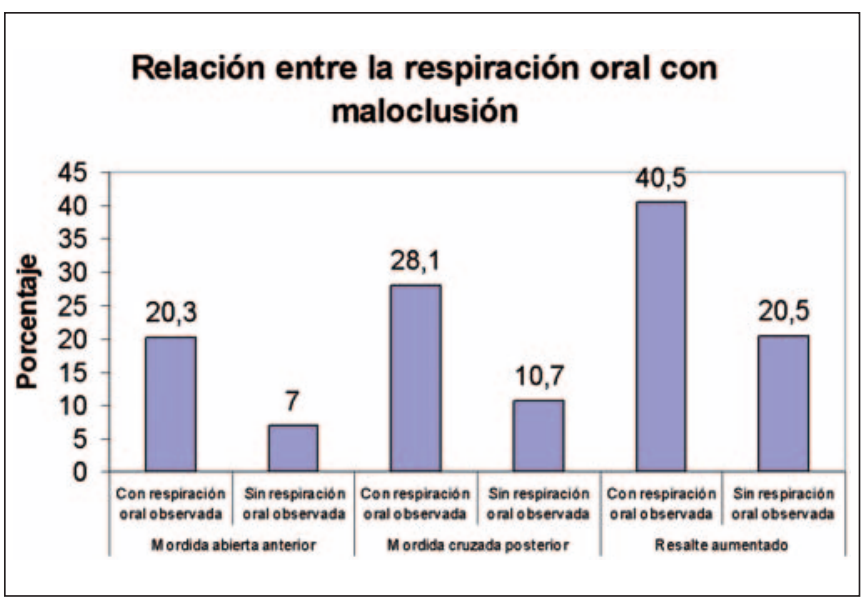

Gráfico 5. 


\section{DISCUSIÓN}

Existe un gran número de estudios que relacionan los distintos hábitos orales, con la presencia de patología oclusal. La mayoría de estos hábitos se producen en los primeros años de vida, así que tiene lógica relacionar estas fuerzas constantes con la deformación del macizo craneomaxilar. El patrón genético es el que da la información definitiva.

Para algunos autores (7), dentro de las maloclusiones, la mordida abierta presenta una prevalencia del $17,7 \%$ en dentición mixta y un $36,3 \%$ de ellas son causadas por patrón genético y hábitos de succión prolongados en el tiempo. En el grupo de niños con mordida abierta anterior, un $63,4 \%$ presentan hábitos de succión y un $61 \%$ patrón facial hiperdivergente.

Estos mismos autores comparan las características oclusales de pacientes con hábito de succión e hiperdivergencia facial con un grupo control de pacientes con hábito de succión y con patrón vertical normal, ambos grupos en la dentición mixta (8).

Warren, en su estudio en niños con dentición temporal defiende que la succión digital y el chupete producen alteraciones oclusales (aumento del resalte y tendencia a mordida abierta anterior) y también que producen tipos de maloclusiones distintas: relaciona el chupete con la mordida cruzada posterior y la succión digital con el aumento del resalte. Sus resultados defienden que hay más prevalencia de mordida cruzada posterior en niños con uso de chupete más de 24 meses, el resalte y mordida abierta aumenta si el hábito dura más de 48 meses y la profundidad palatina a partir de los 36 meses (9).

Emmerich en un trabajo publicado (10), presenta los siguientes porcentajes: el $8,7 \%$ de los niños que nunca usaron el chupete tiene mordida abierta y un $18.7 \%$ resalte aumentado frente al $40,5 \%$ que tiene mordida abierta y $44 \%$ con resalte alto en niños con hábito de chupete.

En este trabajo, dentro del grupo que usó chupete más de 3 años, el 22,5\% presentan mordida abierta anterior y $39,4 \%$ resalte aumentado y el $16,3 \%$ nunca usó el chupete.
Según Davidson, a los 12 meses de vida el $40 \%$ de los niños usa el chupete y el $30 \%$ se chupan el pulgar u otro dedo, en cambio a los 3 años la succión digital ya es más frecuente que el chupete y a los 4 años un $12 \%$ se chupan el dedo frente a un $4 \%$ de los chupetes (11).

En otro estudio de 330 niños de cuatro años en Recife (Brasil) determina que la prevalencia de maloclusión es del $49,7 \%$ y que dentro de ese grupo, un $28,5 \%$ de los niños presentan dos o tres factores que contribuyen a esa maloclusión, siendo el chupete y succión digital unos hábitos directamente relacionados con la maloclusión. Dentro de ese porcentaje de maloclusiones causadas por hábitos, un $12,1 \%$ son mordida cruzada posterior, un $36,4 \%$ de mordida abierta anterior y un $29,7 \%$, resalte aumentado (12).

La prevalencia total de hábitos en los escolares valencianos es del $53 \pm 3 \%$, siendo el $24,28 \%$ niñas y el 28,72 niños y dentro de los niños sin hábitos en porcentaje de niñas es 24,89 y de niños 22,09.

Estos datos muestran que la diferencia estadística no es significativa, concluyendo que es similar sin importar el sexo. En su estudio los hábitos que se presentaron con mayor frecuencia (que se estudien en este trabajo) de mayor a menos frecuencia fueron respiración bucal, succión digital y uso de chupete. Las patologías más frecuentes fueron la mordida abierta y la compresión del maxilar (13).

En sus estudios, Bishara (14), concluye que a finales del primer año de vida el $40 \%$ de los niños/as usan chupete y el $31 \%$ presentan el hábito de la succión digital.

En el segundo año bajan los valores a $25 \%$ y $22 \%$ respectivamente y en el tercer año el $22 \%$ siguen con el chupete y el $14 \%$ con el dedo. A los cuatro años ya se invierten las cifras, siendo significativamente menor el número de niños que usan chupete a los que presentan el hábito de succión digital.

Leite-Cavalcanti (15), estima que entre la edad de 3 a 5 años, la prevalencia de maloclusiones en relación con hábitos no nutritivos es muy alta (87\%), siendo el hábito de chupete de alta prevalencia también $(84,8 \%)$, casi el mismo valor que la succión di- 
gital (84,2\%), y del total de los niños evaluados, el $73,4 \%$ tienen uno o más hábitos no nutritivos. Considera este porcentaje muy alto en comparación con otros estudios en otros países.

Concluye que los niños con hábitos orales tienen 12 veces más peligro de desarrollar maloclusión que los niños sin hábitos.

Karen Glazer Peres (16) sustenta la prevalencia de la maloclusión, en relación con hábitos de succión y con lactancia materna, de un $46,2 \%$ para la mordida abierta y $18,2 \%$ para la mordida cruzada posterior. Relaciona la mordida abierta con el uso del chupete entre los 12 y 48 meses de vida y con el hábito de succión digital hasta los 6 años o más.

Según Helio Scavone-Junior (17) la prevalencia de mordida cruzada posterior en niños y niñas con hábito de chupete es de $11,2 \%$ en aquellos que el hábito persiste hasta los 2 años de edad y 11,4\% en los que se continúa hasta los 6 años.

En su trabajo Olivera Gois (18) concluye que la maloclusión en la dentición primaria está directamente relacionada con el uso del chupete más de 2 años y con la respiración oral. No encuentra asociación significativa entre la succión digital y la maloclusión cuando la succión termine antes de los 2 años. Asegura que los niños con hábitos no nutritivos presentan hasta 6 veces más riesgo de desarrollar una oclusión patológica, y si estos hábitos duran más de 2 años, el peligro asciendo hasta 13,6 veces más.

Otros estudios sostienen que a los 15 meses el 62,3\% de los niños tienen hábitos no nutritivos $(37,6 \%$ chupete y $22 \%$ succión digital) y que a los 36 meses se ha reducido al $40 \%$. Los dos hábitos influyen en el desarrollo de la dentición, sobre todo en la alineación del segmento anterosuperior y en el desarrollo de mordidas abiertas anteriores y cruzadas (19).

Para Romero-Maroto y col. la prevalencia es muy variable, oscilando entre el 1,7 y el $47 \%$, dependiendo de la edad a la que se realice el estudio, pues mientras en las primeras semanas de vida este hábito es muy frecuente al responder a un reflejo innato, conforme el niño va creciendo la frecuencia disminuye.
Por otro lado sostienen que es a partir de los 4 años cuando la persistencia del hábito influye más negativamente en el desarrollo originando maloclusiones en cambio yo me sustento en la teoría de que ya es a partir de los 4 años.

Aseguran que estas maloclusiones no se producen exclusivamente por la presión directa que sobre las arcadas ejerce el dedo, sino que su desarrollo también se ve favorecido por la modificación del funcionamiento de la musculatura perioral y lingual que provoca el hábito (20).

\section{CONCLUSIONES Y RECOMENDACIONES}

Los hábitos orales anómalos modifican la posición de los dientes y la relación y la forma que guardan las arcadas dentarias entre sí, interfiriendo en el crecimiento normal y en la función de la musculatura orofacial.

La prevalencia de estos hábitos depende de la edad del niño, en edades entre 2-4 años el chupete está ampliamente extendido, mientras que en los niños mayores de 4 años lo es la respiración oral. La succión digital tiene una prevalencia mucho menor, pero a pesar de ser menor frecuente persiste más en el tiempo y es más difícil de dejar.

El papel del odontólogo y el pediatra son básicos en la prevención de las maloclusiones, ya que si el hábito es retirado a partir de los 3 años se evitarán transformaciones óseas y dentales. Los dos hábitos deben tratarse cuanto antes valorando que vayamos a tener colaboración con el paciente porque si se espera mucho el hábito ya está más instaurado y es más difícil de superar. La detección precoz de las manifestaciones orales de estos hábitos es básica para garantizar un buen pronóstico.

La prevalencia general de maloclusión es del 37,37\%. Se demuestra la relación directa entre los HSNN y la maloclusión porque en las tres patologías oclusales estudiadas hay diferencia estadísticamente significativa $(p<0,05)$. de mayor prevalencia de maloclusión en el grupo con succión digital y/o chupete más de 3 años. La prevalencia final para el grupo con uso de chupete y succión digital igual o mayor a 3 años es de 45,9\%. 
La diferencia entre la maloclusión encontrada en el grupo general y la hallada en el grupo de riesgo (chupete 3 años o más) es estadísticamente significativa $(\mathrm{p}<0,05)$.

Existe relación entre el uso prolongado en el tiempo del chupete y la succión digital con el hábito de respiración oral, ya que en el grupo de chupete y succión digital más de 3 años encontramos más de un $40 \%$ de respiradores orales observados.

La patología más frecuente es el resalte aumentado, seguida de la mordida cruzada posterior y por último la mordida abierta anterior. Es más frecuente la patología oclusal en la mujer tanto en el grupo de patología general como en el grupo de patología asociada a HSNN.

Un dato importante es la diferencia que existe entre los padres que detectan que sus hijos presentan respiración oral $(22,6 \%)$ y la respiración oral detectada en la revisión (41,6\%). Como además la respiración oral está relacionada con la mayor prevalencia de resaltes aumentados, mordidas cruzadas y mordidas abiertas siendo estas diferencias estadísticamente significativas considero que sería necesario recomendar más visitas al otorrinolaringólogo y una mayor coordinación e intercambio de información entre los distintos especialistas (odontopediatras, otorrinolaringólogos y pediatras) implicados en la materia con el fin de evaluar posibles alteraciones de las vías respiratorias.

El hábito que presenta mayor prevalencia es el chupete y el de menor es la succión digital. Si bien hay que destacar que de los 33 casos con succión digital, el $85 \%$ de ellos persiste más de 3 años, por lo que concluyo que aunque es un hábito poco frecuente, la duración es alta, es un hábito difícil de superar por lo que se le debe dar especial énfasis en su prevención.

El hábito del chupete tiene mayor prevalencia en las mujeres durante los 3 primeros años, pero en cambio, a partir de los 3 años ya se igualan en número de casos en ambos sexos. En cambio en el hábito de succión digital, en los todas las edades es mayor en las mujeres, pero incluso es mayor el porcentaje de mujeres en el grupo de edad igual o mayor a 3 años.
La patología oclusal es más frecuente en la mujer, sin ser estadísticamente significativo.

\section{BIBLIOGRAFÍA}

1. Canut JA. Desarrollo de la oclusión. En: Canut JA. Ortodoncia clínica. Salvat. Barcelona 1989.

2. Yamaguchi H, Sueishi H. Malocclusion associated with abnormal posture. Bull Tokyo Dent Coll 2003; 44(2):43-54.

3. Yolota R, Mishiro M, Abe T, Miyake A, Shiin N, SueishiK, Yamaguchi H. Pressure on Anterior Region of Palate during Thumb-Sucking. Bull Tokyo Coll 2007;48(2):57-66.

4. Larsson E. Sucking, Chewing, and Feeding Habits and the Development of Crossbite: A Longitudinal Study of Girls from Birth to 3 Years of Age. Angle Orthod 2000;71(2):116-9.

5. Bishara SE, Larsson E. Dent Assist Finger habits: their effects and their treatments -part 1. Dent Assist 2007;76 (1):14-6.

6. Bishara SE, Larsson E. Finger habits: their effects and their treatments -part 2. Dent Assist 2007;76 (2):16-8.

7. Cozza P, Baccetti , Franchi L, Mucedero M, Polimeni A. Sucking habits and facial hiperdivergency as risk factors for anterior open bite in the mixed dentition. Am J Orthod Dentofacial Orthop 2005;128: 517-9.

8. Cozza P, Baccetti T, Franchi L, Mucedero M, Polimeni A. Tranverse features of subjects with sucking habits and facial hyperdivergency in the mixed dentition. J Orthod Dentofacial Orthop 2007;132: 226-9.

9. Warren JJ, Bishara E. Duration of nutritive and non-nutritive sucking behaviours and their effects on the dental arches in the primary dentition. Am J Orthod Dentofacial Orthop 2002;121: 347-56. 
10. Emmerich A, Fonseca L, Elias A.M, Vieira de Medeiros $U$. The relationship between oral habits, oronasopharyngeal alterations, and malocclusion in preschool children in Vitória, Espírito Santo, Brazil. Cad Saúde Pública Rio de Janeiro 2004; 20 (3):377-89.

11. Davidson L. Thumb and finger sucking. Pediatr in $\operatorname{Rev} 2008 ; 29$ (6):207-8.

12. Tornisiello CR, Rosenblatt A, Costa PP. Nonnutritive sucking habits in Brazilian children: Effects on deciduous dentition and relationship with facial morphology. Am J Orthod Dentofacial Orthop 2004;126:53-7.

13. Paredes A, Paredes C. Prevalencia de los hábitos bucales y alteraciones dentarias en escolares valencianos. An Pediatr (Barc) 2005;62(3): 261-5.

14. Bishara E, Warren JJ, Broffitt B, Levy SM. Changes in the prevalence of nonnutritive sucking patterns in the first 8 years of life. Am $\mathrm{J}$ Orthod Dentofacial Orthop 2006;130:31-6.

15. Leite-Cavalcanti A, Medeiros-Bezerra PK, Moura C. Aleitamento Natural, Aleitamento Artificial, Hábitos de Sucção e Maloclusões em Pré-escolares Brasileiros. Rev Salud Pública Bogotá 2007; 9(2): 194-204.

16. Peres KG, Barros AJ, Peres MA, Vitora CG. Effects of breastfeeding and sucking habits on malocclusion in a birth cohort study. Rev Saúde Pública São Paulo 2007;41(3):343-50.

17. Scavone-Junior H, Ferreira RI, Mendes TE, VelliniFerreira F. Prevalence of posterior crossbite among pacifier users: a study in the deciduous dentition. Braz Oral res São Paulo 2007;21(2): 153-8.

18. Olivera EG, Campos H, Pimenta $M$ y col. Influence of Nonnutritive Sucking Habits, Breathing Pattern and Adenoid Size on the Development of Malocclusion. Angle Orthod 2008;78(4):647-65.

19. Duncan K, McNamara C, Ireland AJ, Sandy JR. Sucking habits in chilhood and the effects on the primary dentition: finding of the Avon Longitudinal Study of Pregnancy and Childhood. Int J Paediatr Dent 2008;18:178-88.

20. Romero-Maroto M, Romero-Otero P, Pardo de Miguel AM, Sáez-López M. Tratamiento de la succión digital en dentición temporal y mixta. RCOE 2004;9(1):77-82.

\section{CORRESPONDENCIA}

Adolfo Pipa Vallejo

Escuela de Odontología

Catedrático José Serrano, s/n

33006 Oviedo

Correo electrónico: pipaadolfo@uniovi.e 